\title{
Ein Ethylen-Meßgerät auf der Basis der Chemilumineszenz
}

\author{
Bernd Stark, Lothar Martini, Roland Wernecke
}

\section{Einleitung}

Ethylen $\left(\mathrm{C}_{2} \mathrm{H}_{4}\right)$ gehört chemisch zu den Alkenen und ist ein farbloses, erdölartig riechendes Gas. Es ist gekennzeichnet durch eine „ungesättigte“ Kohlenstoff-Kohlenstoff-Bindung, die verantwortlich ist für seine hohe Reaktivität.

Ethylen kommt in der Atmosphäre in einem Mischungsverhältnis vor, dessen Bereich sich von der Nachweisgrenze bei ca. 2 ppb in eher ländlichen Gebieten bis zu Spitzenkonzentrationen von mehr als $50 \mathrm{ppb}$ in Stadtkernen erstreckt [1][2].

Seine Herkunft ist sowohl natürlicher als auch anthropogener Natur. Natürlich entsteht Ethylen als Reifegas beim normalen Stoffwechselvorgang in Früchten und Pflanzen. Anthropogen wird es in die Atmosphäre als Hauptkomponente der Abgase von Verbrennungsmotoren emittiert [3].

In der Atmophäre hat das Ethylen einen nicht zu übersehenden Einfluß auf die Erhöhung der Ozonproduktionsrate [4][5]. Die Möglichkeit seiner Messung unter den verschiedenen atmosphärischen Bedingungen in Verbindung mit anderen Komponenten der Nichtmethan-Kohlenwasserstoffe ist deshalb eine wichtige Aufgabe im Rahmen der atmosphärischen Forschung. Wirtschaftlich kommt der Möglichkeit der Messung von Ethylen eine große Bedeutung im Zusammenhang mit dem Transport von Früichten zu, da das beim Stoffwechselvorgang entstehende und frei werdende Ethylen den Reifungsprozeß der Früchte stark beschleunigt und damit einen Vorgang beeinflußt, der während des Transportes nur bedingt erwünscht ist. Bananen z.B., die mit ca. 10,5 Millionen Tonnen pro Jahr etwa 25 - 30\% der weltweiten Kühlschiffsladung ausmachen, werden im grünen Zustand gepfluickt und mit Ethylen gereift, sollen jedoch während des Transportes vom Ursprungsland zum Verbraucherland weitgehend grün bleiben. Da aber auch grüne Bananen während des Transportes dem Stoffwechsel unterliegen und Ethylen erzeugen, ist eine Kühlung zur Minderung des natuirlichen Stoffwechsels und damit zur Herabsetzung der Reifung und Alterung des transportierten Gutes notwendig. Zum Problem der Kühlung des Fruchtgutes hinzu kommt die Notwendigkeit der Luftspiilung oder der Ethylenabsorption im Transportcontainer im Sinne einer Ethylenminderung. Ethylenabsorber arbeiten nach dem Prinzip der katalytischen Verbrennung, die sehr energieintensiv ist. Daher sollte dieser Betrieb abhängig vom Ethylengehalt im Transportcontainer erfolgen. Das jedoch erfordert eine Ethylenmessung unter betriebsmäßigen Bedingungen auf Kühl- und Kühlcontainerschiffen, die zum gegenwärtigen Zeitpunkt noch nicht durchgefuihrt wird, da geeignete Ethylenmeßsysteme bisher nicht zur Verfügung gestanden haben.

\section{Problemdarstellung}

Das Problem des optimalen Transportes von Südfruichten auf Schiffen ist eine Frage der Bereitstellung einer geeigneten Transportatmosphäre (controlled atmosphere-CA) auf Kuihl- und Kühlcontainerschiffen. Das wiederum ist mit sehr energieintensiven Maßnahmen verbunden und damit eine Frage der Wirtschaftlichkeit [6].

Um einen optimalen Transport der Früchte über einen längeren Zeitraum zu gewährleisten, ist eine kontinuierliche bis quasi kontinuierliche Messung der Ethylenkonzentration der Kühlcontainerluft bzw. der Atmosphäre in den Kühlschiffen im Sinne einer optimalen Einstellung des Ethylengehaltes der Luft erforderlich. Dazu ist eine Meßtechnik nötig, die kontinuierlich im Spurenbereich Meßdaten liefert. Diese Meßtechnik muß außerdem robust und unter extremen Bedingungen, wie z.B. hohen Temperaturen und hoher Luftfeuche, einsatzfähig und dazu von Personal bedienbar sein, das nicht für den Umgang mit dieser Meßtechnik ausgebildet ist. Eine weitere wesentliche Forderung an die Meßtechnik besteht darin, daß das Gerät auch über einen längeren Zeitraum wartungsfrei und zuverlässig im Rahmen der geforderten Genauigkeit funktioniert. In der vorliegenden Arbeit wird ein Ethylen-Meßgerät vorgestellt, das auf dem Prinzip der Chemilumineszenz beruht und selektiv und kontinuierlich die Messung der Ethylen-Konzentration gestattet. Dazu ist dieses Gerät weitgehend den auf Kühlschiffen herrschenden Bedingungen angepaßt.

\section{Nachweismethoden für Ethylen im Spurenbereich}

Die Messung von $\mathrm{C}_{2} \mathrm{H}_{4}$ im extremen Spurenbereich von 1 - $50 \mathrm{ppb}$ ist meßtechnisch sehr aufwendig und mit hohen Kosten verbunden. Mit den gegenwärtig zur Verfügung stehenden Meßmethoden ist dieser Konzentrationsbereich zugänglich, jedoch nur für die atmosphärische Forschung relevant.

Die angewandten spektroskopischen Methoden nutzen die Absorptionsbande von $\mathrm{C}_{2} \mathrm{H}_{4}$ im mittleren IR-Bereich bei $10,5 \mu \mathrm{m}\left(950 \mathrm{~cm}^{-1}\right)$, um mit Langwegmessungen oder mit Hilfe von Langweg-Multireflexionszellen $\mathrm{C}_{2} \mathrm{H}_{4}$ in der Atmosphäre zu bestimmen. In der Abb. 1 ist in einem Absorptionsspektrum von Luftschadstoffen die IR-Ethylenbande deutlich ausgeprägt [7]. Als Meßmethoden kommen dabei hauptsächlich die Absorptionsspektroskopie mit durchstimmbaren Diodenlasern (TDLAS) [8] und die Fourier-Transformationsspektroskopie im IR (FTIR) [9] zur Anwendung. Beide Methoden haben eine Nachweisgrenze von etwa $1 \mathrm{ppb}$. 


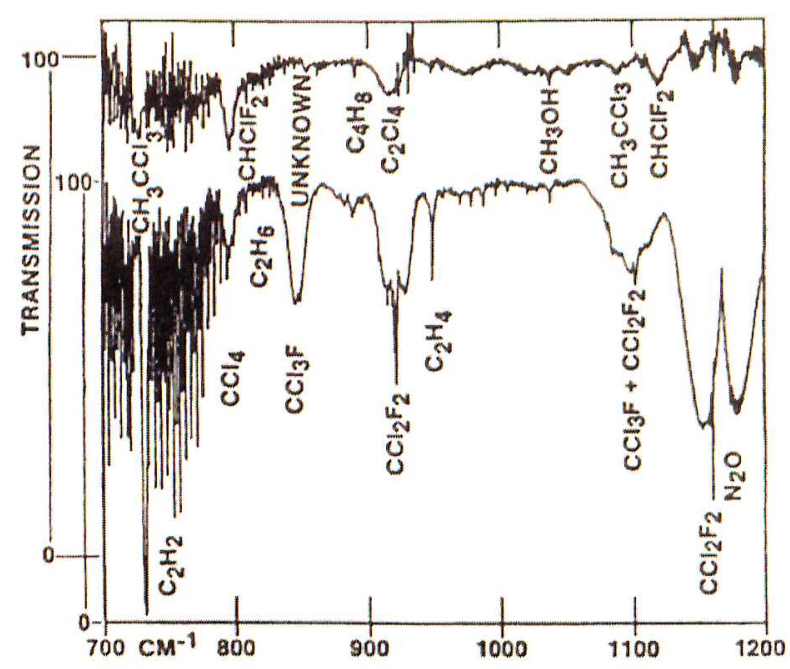

Abb.1: Absorptionsspektrum von Luftschadstoffen mit deutlich ausgeprägter Absorption von Ethylen $\left(\mathrm{C}_{2} \mathrm{H}_{4}\right)$ bei $950 \mathrm{~cm}^{-1}[7]$

Als weitere spektroskopische Methode ist fuir Bestimmungen von Ethylen das Differentielle Absorptionslidar (DIAL) zur Anwendung gekommen [10]. Die Nachweisgrenze beträgt $16 \mathrm{ppb}$ [10]. Die Methode ist jedoch als „remote sensing"-Methode nur fuir atmosphärische Anwendungen geeignet und zudem extrem aufwendig und teuer.

Mit der Methode der Photoakustischen Spektroskopie (PAS), auch mit Photoakustik oder Optoakustik bezeichnet, wurden Konzentrationen unter 1 ppb nachgewiesen [11]. Allerdings ist auch diese Methode experimentell sehr aufwendig und für Messungen unter extremen Bedingungen nicht geeignet.

Die Methode der Gaschromatographie in Verbindung mit einem geeigneten Detektor ist für die Bestimmung von Ethylen in der Atmosphäre bei ausreichender Anreicherung und richtiger Aufgabe der angereicherten Probe auf die Trennsäule die Methode mit der niedrigsten Nachweisgrenze im sub-ppb-Bereich. Es muß jedoch darauf hingewiesen werden, daß die Anwendung der Gaschromatographie sehr viel Erfahrung des Experimentators voraussetzt und praktisch nicht anwendbar ist für Personal, das für Arbeiten mit Gaschromatographen nicht geschult ist. Hinzu kommt, daß kommerzielle Gaschromatographen sehr teuer in der Anschaffung und in der Unterhaltung sind. Die Analyse der Proben mit einem befriedigenden Resultat kann nur über Probenahme vor Ort mit anschließender Analyse im Labor erfolgen. Damit ist diese, als Standardmethode zur Bestimmung von Kohlenwasserstoffen anerkannte Meßmethode für die in dieser Arbeit dargestellte Problematik nicht anwendbar.

Die Verwendung der UV-Absorption von Ethylen bei Wellenlängen im Bereich 200 - $220 \mathrm{~nm}$ zur Bestimmung von $\mathrm{C}_{2} \mathrm{H}_{4}$ ist zwar prinzipiell möglich, erfordert aber bei der geringen Größe der Absorption und der notwendigen kleinen Bandbreite des Meßbereiches eine aufwendige und diffiziele Meßtechnik.

\section{Meßmethode}

In dem für die spezifische Ethylenbestimmung entwickelten Meßgerät kommt die Methode der homogenen Gasphasen-Chemilumineszenz zur Anwendung [12].
Diese Methode ist in der analytischen Chemie ein relativ junges Verfahren. Die Zahl der Reaktionen, die Chemilumineszenz zeigen, ist relativ klein, so daß die Zahl der Gase, die mit dieser Methode nachweisbar ist, stark eingeschränkt ist. Die Methode ist jedoch trotz ihrer Einschränkung von großer Bedeutung für die Analytik, da eine Reihe von Stoffen, die mit Hilfe der Chemilumineszenz direkt oder indirekt bestimmbar sind, in der Umwelt eine große Rolle spielen. Zu diesen Stoffen zählen z.B. Ozon, Stickoxide, Ammoniak, Olefine und organische Sulfide [13].

\subsection{Physikalische Grundlagen}

Reagieren Stoffe chemisch miteinander, so kann sich, wenn diese Reaktion hinreichend exotherm abläuft, die freiwerdende Energie bei einem oder mehreren der Reaktionsprodukte anstelle von Wärmeenergie als elektronische Anregungsenergie, Schwingungsenergie oder Rotationsenergie äußern. Der Übergang des angeregten Stoffes in den Grundzustand ist dann mit der Emission von Strahlung verbunden. Dieser Vorgang wird wegen der chemischen Quelle der Energie mit Chemilumineszenz bezeichnet. Da die Menge des entstehenden Lichtes proportional zur Zahl der angeregten Reaktionsprodukte ist, wird das Licht quantitativ als Maß für die Bestimmung der Konzentration eines Stoffes benutzt.

Als Folge der Reaktion zwischen Ozon und Ethylen entsteht angeregtes Formaldehyd, das beim Übergang in den Grundzustand Strahlung emittiert [14][15]:

$\mathrm{O}_{3}+\mathrm{C}_{2} \mathrm{H}_{4} \rightarrow \mathrm{HCHO}^{*}+$ andere Produkte

$\mathrm{HCHO}^{*} \rightarrow \mathrm{HCHO}+\mathrm{hv}(300 \leq \lambda \leq 550 \mathrm{~nm})$

Die spektrale Strahlungsverteilung ist in Abb. 2 dargestellt [15].

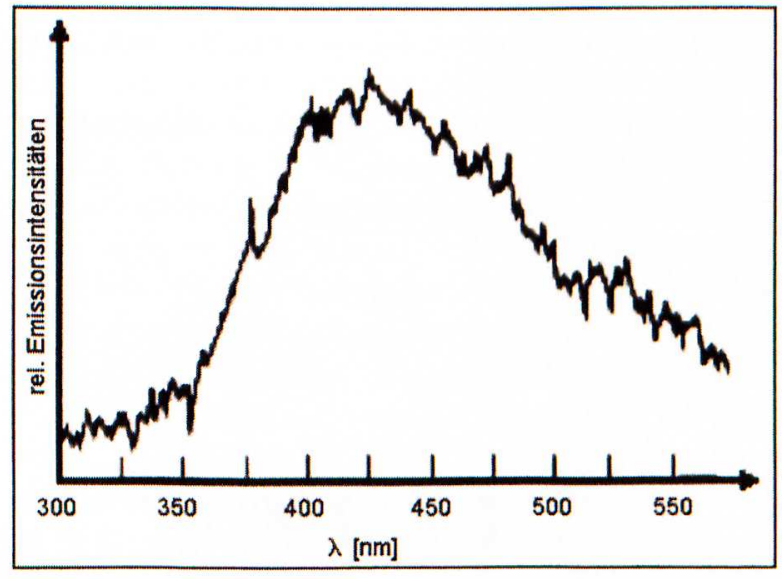

Abb. 2: Spektrale Strahlungsverteilung der Chemilumineszenz-Strahlung von angeregtem Formaldehyd [15]

Der Vorteil dieser Reaktion ist, daß sie unter Normaldruck abläuft. Im Gegensatz dazu ist die Reaktion zwischen Ozon und anderen Olefinen nur bei niedrigeren Drücken analytisch relevant, da unter Normaldruck die elektronisch angeregten Zustände gelöscht werden [16]. Praktisch bedeutet das fuir den in dieser Arbeit beschrie- 
benen Ethylenanalysator, daß unter Normaldruckbedingungen gearbeitet werden kann und dabei entstehende Reaktionsprodukte möglicher anderer Olefine unwirksam sind.

Die Reaktion (4.1) läßt sich sowohl zur Bestimmung von Ozon als auch von Ethylen verwenden [15]. Wichtig für die Bestimmung von Ethylen ist nur, daß in den Reaktionsraum Ozon im Überschuß eingeführt wird. Ist dieser Sachverhalt gewährleistet, stellt die entstehende Strahlungsmenge ein Maß für die vorhandene Ethylenkonzentration dar.

Im Beisein von Stickoxid (NO) läuft zusätzlich die folgende chemilumineszente Reaktion ab [15]:

$$
\begin{aligned}
& \mathrm{O}_{3}+\mathrm{NO} \rightarrow \mathrm{NO}_{2}{ }^{*}+\mathrm{O}_{2} \\
& \mathrm{NO}_{2}{ }^{*} \rightarrow \mathrm{NO}_{2}+\mathrm{hv}(590 \leq \lambda 3000 \mathrm{~nm})
\end{aligned}
$$

Da sich der nach Reaktion (4.4) entstehende Spektralbereich der Chemilumineszenzstrahlung ohne Überlappung an den nach Reaktion (4.2) entstehenden Spektralbereich anschließt, ist es praktisch unproblematisch, durch Wahl eines geeigneten Filters die nach Reaktion (4.4) entstehende Strahlung gegenuiber der Meßstrahlung abzublocken.

\section{Meßapparatur}

Der Vorteil der Methode der homogenen GasphasenChemilumineszenz liegt darin, daß der notwendige apparative Aufwand gegenuiber anderen analytischen Methoden vergleichsweise klein gehalten werden kann. Der hier beschriebene Ethylenanalysator besteht in seinen wesentlichen Elementen aus

- der Reaktionskammer

- dem Strahlungsempfänger

- dem Ozongenerator

- der Pumpe

- dem Ozonscrubber

- dem Elektronikteil

Das Gesamtschema des Analysators zeigt die Abb. 3.

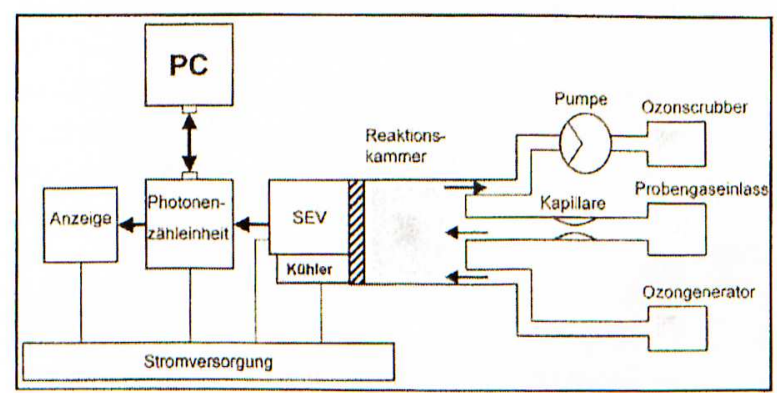

Abb. 3: Schematischer Aufbau des Ethylen-Analysators

\subsection{Funktionsweise des Analysators}

Die homogene Gasphasenreaktion zwischen dem Ethylen enthaltenden Meßgas und dem Ozon findet in der Reaktionskammer statt. Die bei der Reaktion entstehende chemilumineszente Strahlung wird durch einen
Photomultiplier (SEV) gemessen, der an der Reaktionskammer angeflanscht ist. Die durch den Photomultiplier gemessene Strahlung ist ein Maß fuir die im Meßgas enthaltene Ethylenkonzentration. Die Möglichkeit der Angabe der Ethylenkonzentration erfolgt über eine Kalibration. Die Kalibration wird mit Hilfe eines Kalibrationsgases bekannter Ethylenkonzentration und der Zuordnung des zugehörigen Meßsignals durchgefuihrt. Der Meßgasstrom und der das Ozon enthaltende Reaktionsgasstrom durch die Reaktionskammer werden durch eine Gaspumpe angetrieben. Die eingestellte durchfließende Meßgasmenge beträgt $0,3 \mathrm{l} / \mathrm{min}$. Für diese Gasmenge ist ein hinreichend hohes Ozonmischungsverhältnis von $>5000$ ppm gegenüber der zu messenden Ethylenkonzentration von 1 - 10 ppm gewährleistet. Das Meßsystem wird bei Normaldruck betrieben. Der Abgasstrom wird über einen Ozonscrubber geleitet, bevor er an die umgebende Atmosphäre abgegeben wird. Auf diese Weise wird das uiberschüssige Ozon abgebaut, so daß die an die umgebende Atmosphäre abgegebene Abluft ozonfrei ist.

\subsection{Reaktionskammer und Strahlungsempfänger}

Die Reaktionskammer und der Strahlungsempfänger bilden eine Baueinheit in der Weise, daß der Strahlungsempfänger lichtdicht an der Reaktionskammer angeflanscht ist. Die flache zylinderförmige Reaktionskammer hat einen Durchmesser von $4 \mathrm{~cm}$ und ein Volumen

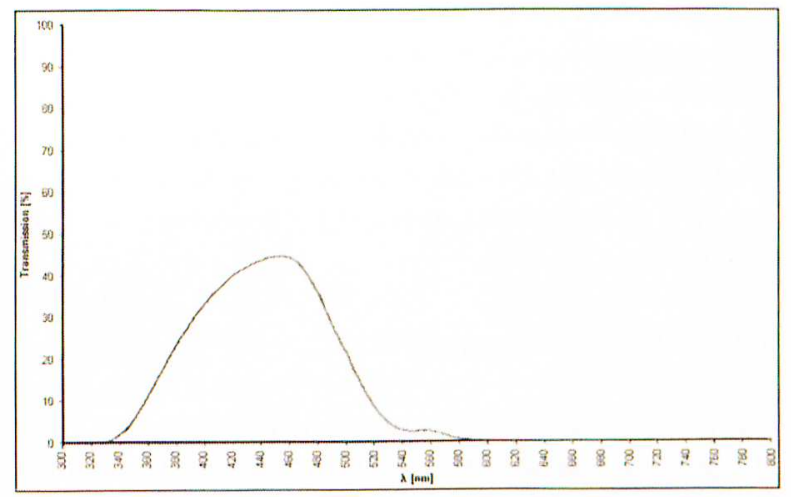

Abb. 4: Transmissionscharakteristik des Strahlungsfilters zwischen Reaktionskammer und Photomultiplier

von $12,5 \mathrm{~cm}^{3}$. Die Reaktionskammer und der Strahlungsempfänger sind durch ein Fenster getrennt. Dieses Fenster ist eine Kombination aus einer geeigneten Kunststoffscheibe, die vakuumdicht in die Reaktionskammer geklebt ist, und einem BG-28-Filter. Diese Kombination stellt ein Strahlungsfilter dar, dessen Transmissionscharakteristik in der Abb. 4 dargestellt ist. Strahlung der Wellenlänge $\lambda \geq 580 \mathrm{~nm}$ kann dieses Filter nicht passieren, wodurch gewährleistet ist, daß Lumineszenzstrahlung aus der möglichen Reaktion zwischen Ozon und Stickoxid, die das Meßergebnis verfälschen könnte, nicht auf den Strahlungsempfänger fallen kann.

Als Strahlungsempfänger wird entsprechend der Strahlungsverteilung der Lumineszenzstrahlung ein blauempfindlicher Photomultiplier Typ R6095P der Fa.Hamamatsu verwendet, der sich bei der gewählten Saugspannung von -1000 Volt durch eine sehr niedrige 
Dunkelzählrate von nur 10 Impulsen/s auszeichnet. Seine spektrale Empfindlichkeitsverteilung erstreckt sich uiber den Bereich von 300 bis $650 \mathrm{~nm}$. Das Empfindlichkeitsmaximum zwischen 390 und $420 \mathrm{~nm}$ liegt dicht beim Maximum der emittierten Lumineszenzstrahlung von $430 \mathrm{~nm}$ und dem Maximum der Transmissionskurve des verwendeten Strahlungsfilters (Abb. 4).

Die Kombination aus der spektralen Empfindlichkeitsverteilung des Photomultipliers und der Transmissionskurve des Strahlungsfilters bewirkt eine hohe Effektivität des Strahlungsempfängers gegenüber der Lumineszenzstrahlung und damit eine erhöhte Empfindlichkeit.

\subsection{Ozongenerator}

Der Ozongenerator stellt die für die homogene Gasreaktion zwischen dem zu messenden Ethylen und dem Reaktionsgas Ozon hinreichend große Ozongasmenge zur Verfügung. Es handelt sich hierbei um den Ozonator 240.0006/30 der Fa. Eco Physics. Füir den Ozonator kann sowohl trockene Luft als auch Sauerstoff als Versorgungsgas verwendet werden. Im Ozonator wird der molekulare Sauerstoff in einem Bereich sehr hoher Feldstärke dissoziiert. Der entstehende atomare Sauerstoff reagiert mit dem im Versorgungsgas vorhandenen molekularen Sauerstoff unter Bildung von Ozon. Der Gasstrom durch den Ozonator ist mit $0,3 \mathrm{l} / \mathrm{min}$ so gewählt, daß sich ein Ozonmischungsverhältnis von $5,2 \times 10^{-3}$ $(\triangle 0,52 \%)$ einstellt. Damit ist das Verhältnis zwischen dem Ozon und der nachzuweisenden Ethylenmenge sehr groß (ungefähr 103). Schwankungen in der Ozonkonzentration, hervorgerufen durch Schwankungen in der Gasdurchflußmenge bis zu $\pm 15 \%$, sind so ohne Einfluß auf das Meßergebnis. Der Ozonator wird mit einer Spannung von $20 \mathrm{~V}$ bei einem Strom von 0,3 A betrieben.

\subsection{Pumpe und Ozonscrubber}

Die Pumpe und der Ozonscrubber befinden sich am Ausgang des Meßgerätes. In dieser Anordnung wird der Meßgasstrom durch das Meßgerät gesaugt. So wird verhindert, daß sich in der Reaktionskammer ein Druck aufbaut, der die chemische Reaktion beeinflussen könnte und das Meßgas durch eventuelle Emissionen aus der Pumpe verschmutzt wird. Die Förderleistung der Pumpe ist so eingestellt, daß der bereits erwähnte Meßgasstrom von $0,3 \mathrm{l} / \mathrm{min}$ aufrechterhalten wird. Vor dem Verlassen des Gerätes wird der Meßgasstrom durch den Ozonscrubber geleitet, in dem iiber erhitztem Molybdänblech das noch vorhandene Restozon abgebaut wird.

\subsection{Elektronikteil}

Der Elektronikteil hat die Aufgabe

- die notwendigen Versorgungsspannungen zur Verfügung zu stellen

- die vom Photomultiplier kommenden Meßsignale zu verstärken und $\mathrm{zu}$ formen

- die geformten Meßdaten zu verarbeiten

\subsubsection{Hochspannungsversorgungseinheit für Photomultiplier}

Die Hochspannung für den Betrieb des Photomultipliers wird uiber ein einstellbares Hochspannungsmodul Model C4710-01 der Fa. Hamamatsu Photonics bereitgestellt. Dieses Modul wird mit einer Eingangsspannung von +12 Vdc betrieben und liefert eine lineare Ausgangsspannung zwischen $-240 \mathrm{Vdc}$ und $-1500 \mathrm{Vdc}$, die über eine Hilfsspannung 0 bis $+5 \mathrm{Vdc}$ einstellbar ist. Der an das Hochspannungsmodul angeschlossene Lastwiderstand ist so dimensioniert, daß der Grenzwert des Ausgangsstromes von $1 \mathrm{~mA}$ nicht iiberschritten wird.

\subsubsection{Photonenzähleinheit}

Der Photomultiplier wird im sogenannten Zählmodus betrieben, in dem die Zahl der Ausgangsimpulse proportional zur Intensität der Chemilumineszenzstrahlung ist. Diese Betriebsart wurde gewählt, weil sie auch bei sehr niedriger Intensität sehr gute Strahlungsmessungen bei einem günstigen Signal-Rausch-Verhältnis (S/N-ratio) und hoher Stabilität zuläßt.

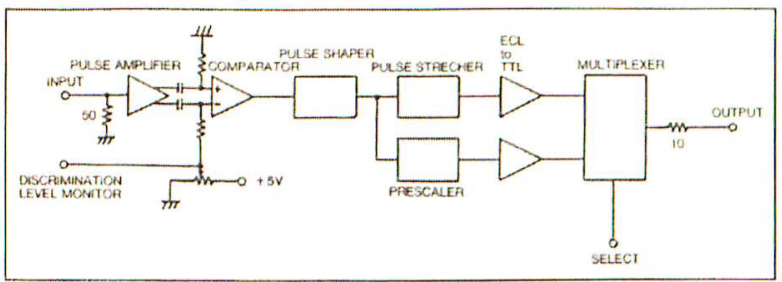

Abb. 5: Signalkette innerhalb der Photonenzähleinheit [17]

In der Abb. 5 ist die Signalkette dargestellt [17]. Danach werden in der Zähleinheit die vom Photomultiplier kommenden Signalimpulse durch einen Impulsverstärker/ Diskriminator auf ein 5V Digitalsignal konvertiert und anschließend einem Impulszähler zugefuihrt. Der Zähler wird mit Hilfe eines PC programmiert. Die vom Zähler kommenden Daten werden im PC unter Berücksichtigung der Kalibrationsdaten weiterverarbeitet. Als Steuerungsprogramm wird LabVIEW verwendet.

Als Photonenzähleinheit wird der Modul C3866 der Fa. Hamamatsu Photonics verwendet, der mit einer Versorgungsspannung von $\pm 5 \mathrm{~V}$ betrieben wird. Da dieser Modul einen Hochgeschwindigkeitsschaltkreis verwendet, gibt es quasi keine Impulsüberlappung im Ausgangsbereich bis zu einer Zählrate von $10^{7}$ counts/s. Auf diese Weise ist Photometrie mit hoher Genauigkeit in einem großen Dynamikbereich möglich. Als Zähler wird der programmierbare Universalzähler HM 8122 mit RS 232 Schnittstelle HO89 der Fa. HAMEG zum PC verwendet.

\section{Kalibration des Gerätes}

Eine Kalibration des Gerätes ist nötig, da eine Zuordnung von zu messender Ethylenkonzentration und Meßwert durch geräteunabhängige Größen nicht möglich ist. Durch die Kalibration erfolgt eine Zuordnung zwischen bekannter, vorgegebener Ethylenkonzentration 


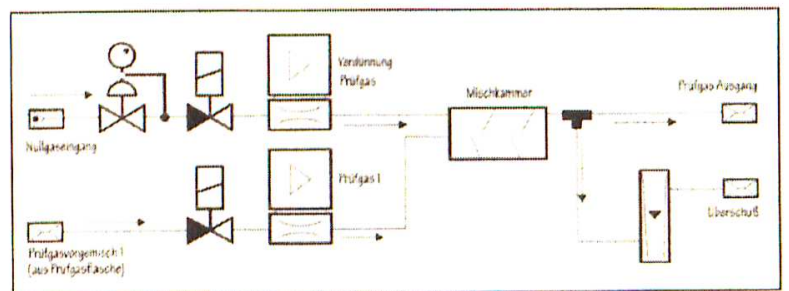

Abb. 6: Gasmischsystem für die Herstellung von Kalibriergas [18]

und zugehörigem Meßwert in Form der Impulszählrate in counts/s.

Das Kalibrationsgas mit bekannter Ethylenkonzentration wird über das Kalibriersystem MK5 der Fa. MCZ Umwelttechnik bereitgestellt. Die Abb. 6 zeigt den Flußplan des Kalibriersystems für die Kombination von einem Prüfgaseingang mit einem Verduinnungsgasfluß [18]. Als Verdünnungsgas wird Nulluft aus einem Nulluftgenerator oder aus einer Gasflasche, als Prüfgas ein Prüfgasvorgemisch aus einer Pruifgasflasche verwendet. Nulluft- und Prüfgasmenge werden über Massenflußregler eingestellt und in einer Mischkammer gemischt. Das Kalibrationsgas wird dann dem Meßgerät über den Pruifgas-Ausgang in hinreichender Menge drucklos zugeführt. Die Berechnung der Konzentration der Gasmischung erfolgt nach der Gleichung:

$c^{\prime}=\frac{c_{x} \times V_{P x}}{V_{V}+V_{P x}}$

$c^{\prime}=$ Konzentration des Kalibrationsgases

$c_{\mathrm{x}}=$ Konzentration des Prüfgasvorgemisches

$\mathrm{V}_{\mathrm{PX}}=$ Volumenstrom des Prüfgasvorgemisches

$V_{V}=$ Volumenstrom des Verdünnungsgases

Das System gestattet die Bereitstellung des Kalibrationsgases in einem Mischungsverhältnisbereich von 10 ppb bis 100 ppm mit einer Genauigkeit von $\pm 1 \%$ und einer Wiederholungsgenauigkeit von $0,25 \%$.

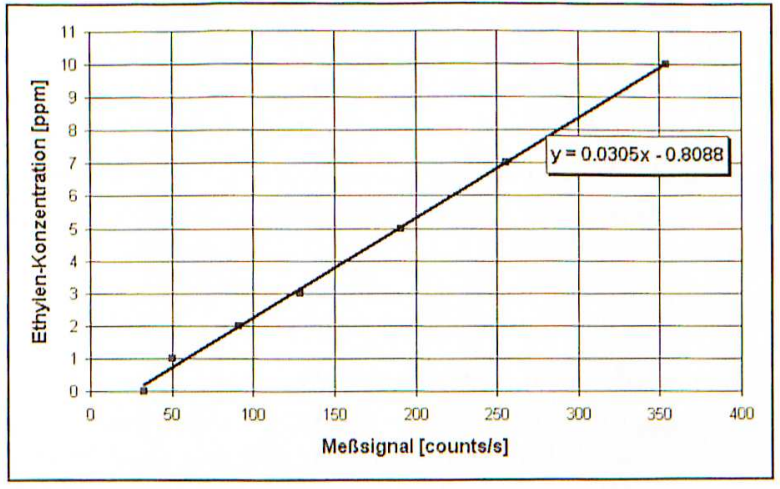

Abb. 7: Kalibrationskurve zur Bestimmung der Kalibrationskonstanten (zugehörige Gerätedaten: Photomultiplierspannung -1000 V, Diskriminatorspannung $800 \mathrm{mV}$, Gasfluß 0,3 l/min)

Für den Konzentrationsbereich von $1 \mathrm{ppm}$ bis $10 \mathrm{ppm}$, dem für die Anwendung des Gerätes relevanten Konzentrationsbereich, ist in der Abb. 7 eine Kalibrationskurve dargestellt. Sie zeigt sehr deutlich den linearen Zusammenhang zwischen der Ethylenkonzentration und dem Meßsignal. Aus der Kalibrationskurve sind durch eine lineare Regression problemlos die beiden
Konstanten bestimmbar, die eindeutig die Kalibrationsgerade festlegen. Mit Hilfe dieser beiden Konstanten, die in den Rechner eingegeben und bis zu einer möglichen Änderung gespeichert werden, wird aus dem gemessenen Signal die Ethylenkonzentration bestimmt.

\section{Meßergebnisse}

Die Leistungsfähigkeit des Meßgerätes wurde getestet, indem es mit einem Meßgas in Form von Gasgemischen aus Ethylen und Nullgas mit unterschiedlicher Ethylenkonzentration beaufschlagt wurde. Das Meßgas wurde mit Hilfe des Kalibriersystems MK5 hergestellt. Die Berechnung der Meßgaskonzentration erfolgte nach Gleichung (6.1). Als Kalibration wurde die in der Abb. 7 dargestellte Kalibrationskurve zugrunde gelegt. Die Messungen zum Vergleich zwischen vorgegebener Meßgaskonzentration und gemessener Konzentration wurden bei einer Photomultiplier-Spannung von $-1000 \mathrm{~V}$ durchgefuihrt. Die eingestellte Diskriminator-Spannung betrug $800 \mathrm{mV}$.

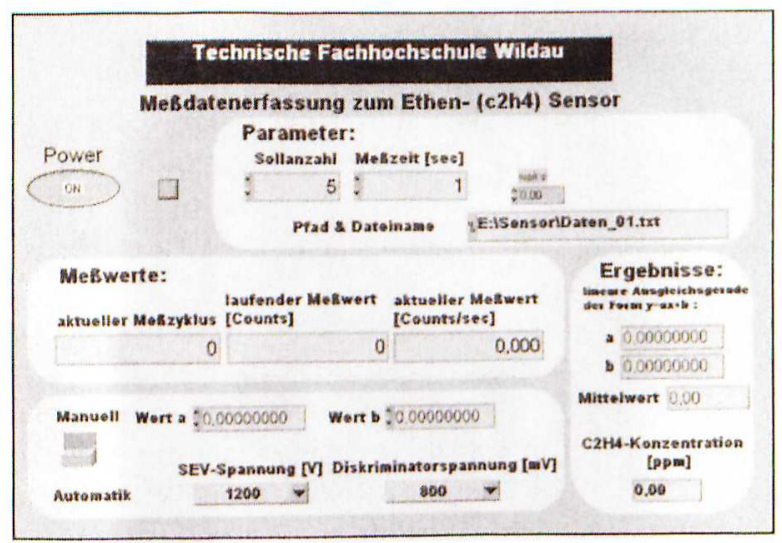

Abb. 8: Benutzeroberfläche des Meßprogramms

In der Abb. 8 ist die Benutzeroberfläche des Meßprogramms dargestellt. Die Einstellung der Meßparameter, die Meßdatenerfassung und die Bereitstellung der gemessenen Konzentrationswerte erfolgt über dieses Meßprogramm.

\begin{tabular}{|c|c|}
\hline $\begin{array}{c}\text { vorgegebene } \\
\mathrm{C}_{2} \mathrm{H}_{4}-\text { Konz. } \\
{[\mathrm{ppm}]}\end{array}$ & $\begin{array}{c}\text { gemessene } \\
\mathrm{C}_{2} \mathrm{H}_{4}-\text { Konz. } \\
{[\mathrm{ppm}]}\end{array}$ \\
\hline 0 & 0,127 \\
\hline 1 & 0,928 \\
\hline 3 & 2,990 \\
\hline 5 & 5,103 \\
\hline 7 & 7.152 \\
\hline 10 & 10,224 \\
\hline
\end{tabular}

Tabelle 1: Vorgegebene und gemessene Ethylen-Konzentration

Die Meßdaten zur Bestimmung der Ethylenkonzentration werden mit einer Frequenz von $1 \mathrm{~Hz}$ bereitgestellt. Die Meßzeit ist einstellbar zwischen $1 \mathrm{~s}$ und offener oberer Grenze. Für die hier präsentierten Messungen wurde eine Integrationszeit von 30 s gewählt. Die Ausgabe der Meßwerte ist über den Bildschirm und in eine Datei möglich. Der Vergleich von vorgegebener und gemessener Ethylenkonzentration ist in der Tabelle 1 dargestellt. In der Abb. 9 sind die Daten der Tabelle 1 mit Regressionsgerade und analytischem Ausdruck graphisch dargestellt. Dem analytischen Ausdruck für die Regressionsgerade ist zu entnehmen, daß über den dargestellten 


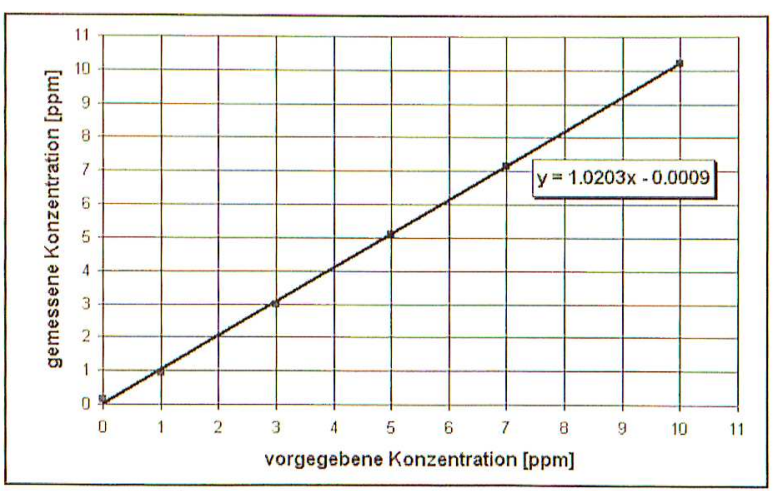

Abb. 9: Graphischer Vergleich zwischen vorgegebener und gemessener Ethylen-Konzentration

Konzentrationsbereich mit einem Fehler von $2 \% \mathrm{zu}$ rechnen ist. Bei Kenntnis dieses Fehlers können so die gemessenen Konzentrationswerte unproblematisch uiber die Kalibration korrigiert werden. Die Abb. 10 zeigt beispielhaft einen Ausschnitt aus einem Stabilitätstest des Gerätes nach einer Einlaufzeit von 1 Stunde ohne Thermostatisierungsmaßnahmen. Entsprechend dem Anstieg der Regressionsgeraden ist eine Kalibration/24 Stunden Laufzeit des Gerätes ausreichend. Die Stabilität des Gerätes ist dabei sehr wahrscheinlich noch durch Thermostatisierung der Reaktionskammer und Kühlung des Photomultipliers mit Hilfe eines Peltier-Küihlers zu verbessern.

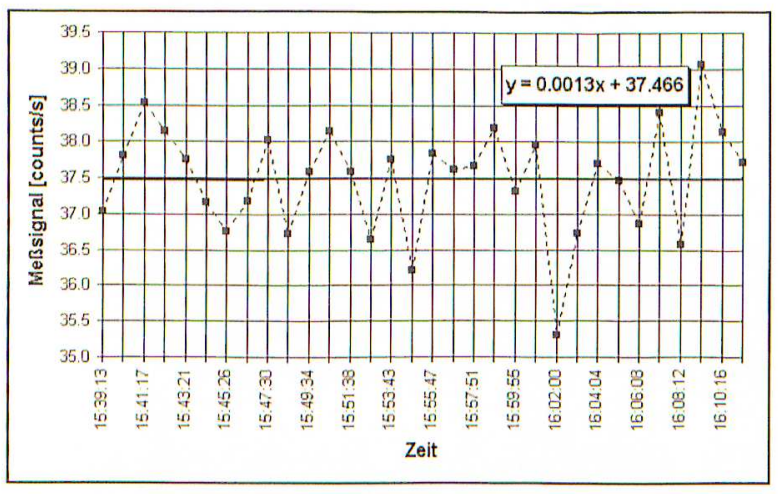

Abb. 10: Stabilitätstest des Ethylen-Analysators nach einer Einlaufzeit des Gerätes von einer Stunde

\section{Schlußbemerkungen}

Mit dem in der vorliegenden Arbeit vorgestellten Ethylen-Analysator steht ein einfaches, wartungsarmes Meßgerät auf der Basis der Chemilumineszenz zur Verfügung, mit dem spezifisch die Ethylen-Konzentration im oberen ppb- und ppm-Bereich bestimmt werden kann. Das vorgestellte Meßgerät ist besonders für den Einsatz im Friichtetansport geeignet. Es ist sicher möglich, die untere Nachweisgrenze des Gerätes durch Thermostatisierung der Reaktionskammer und Kühlung des Photomultipliers noch herabzusetzen. Dabei sollte ein extrem rauscharmer, angepaßter Photomultiplier und eine sehr rauscharme Elektronik zur Anwendung kommen. Unproblematisch ist der Betrieb des Photomultipliers in der Zählmode. Unter den empfohlenen optimierten Betriebsbedingungen ist es sicher möglich, das Gerät auch im mittleren und unteren ppb-Bereich für Immissionsmessungen zu verwenden.

\section{Literatur}

[1] Air Monitoring by Spectroscopic Techniques; edited by Sigrist, M. W., John Wiley \& Sons, Inc., 1994, p. 216

[2] Meyer, P. L. and Sigrist. M. W.; Atmospheric pollution monitoring using $\mathrm{CO}_{2}$-laser photoacoustic spectroscopy and other techniques. Rev. Sci. Instrum. 61, 1779 - 1807 , (1990)

[3] Air Monitoring by Spectroscopic Techniques; edited by Sigrist, M.W., John Wiley \& Sons, Inc., 1994, p. 205

[4] Crutzen, P. J.; Tropospheric ozone: An overview. In Tropospheric Ozone: Regional and Global Scale Interactions (I.S.A. Isaksen, Ed.), pp. 3 - 32, Reidel, Dordrecht, The Netherlands.

[5] Schurath, U.; Bildung von Photooxidantien durch homogene Transformation von Schadstoffen. In Ges. Strahlen Umweltforsch. Munich, Rep. 17/88, 136 - 151

[6] Verhoeven, B.; Technischer Bericht 01 / 98 Auftrag: 1/3/ 1997, Experimentelle Untersuchungen in Versuchscontainern im Labor des Arbeitsbereiches der TUHH TU Hamburg-Harburg, Arbeitsbereich Hilfsmaschinen und Automation, Neßpriel 5, 21129 Hamburg, 03.02.1998

[7] Hanst, P. L.; Air pollution measurement by Fourier transform spectroscopy. Appl. Opt. 17, 1360 - 1366, (1978)

[8] Partridge, R. H. and Curtis, I. H.; Long-path diode laser measurement of industrial air pollution. In Monitoring of Gaseous Pollutants by Tunable Diode Lasers (R. Grisar, G. Schmidtke, M. Tacke, and G. Restelli, Eds.), pp. 3 - 15, Kluwer Academic, Dordrecht, The Netherlands

[9] Hanst, P. L. and Hanst, S. T.; Gas measurement in the fundamental infrared region. In Air Monitoring by Spectroscopic Techniques (M. W. Sigrist, Ed.), pp. 335 - 470, John Wiley \& Sons, Inc., 1994

[10] Svanberg, S.; Differential Absorption Lidar (DIAL). In Air Monitoring by Spectroscopic Techniques (M. W. Sigrist, Ed.), pp. 139-140, John Wiley \& Sons, Inc., 1994

[11] Sigrist, M. W.; Air Monitoring by Laser Photoacoustic Spectroscopy. In Air Monitoring by Spectroscopic Techniques (M. W. Sigrist, Ed.), pp. 196 - 197, John Wiley \& Sons, Inc., 1994

[12] Skoog, D. A. und Leary, J. J.; Instrumentelle Analytik (Grundlagen-Geräte-Anwendungen), S. 189 - 211 SpringerVerlag, Berlin Heidelberg, 1996

[13] Kummer, W. A., Pitts, Jr., J. N. and Steer, R. P.; Chemiluminescent Reactions of Ozone with Olefines and Sulfides. Environ. Science \& Technology 5, 1045 (1971)

[14] Warren,G. J. and Babcock, G.; Portable Ethylene Chemiluminescence Ozone Monitor. Rev. Sci. Instrum. 41, 280 (1970)

[15] Finlayson-Pitts, B. J. and Pitts, Jr., J. N.; Spectroscopic Monitoring Techniques: Emission Spectrometry. In Atmospheric Chemistry: Fundamentals and Experimental Techniques, pp. 310 - 312, John Wiley \& Sons, Inc., 1986

[16] Finlayson, B. J., Pitts, Jr., J. N. and Atkinson, R.; LowPressure Gas-Phase Ozone-Olefin Reactions. Chemiluminescence, Kinetics, and Mechanisms. J. Am. Chem. Soc. /96: 17/, pp. 5356-5367, 1974

[17] Firmenschrift Hamamatsu, How To Use Photon Counting Unit C3866, Operation Manual, p. 12

[18] Bedienungsanleitung Kalibriersystem MK5/MK10; TDPRZ/MK10 Universal/Version 280797, Umwelttechnik MCZ $\mathrm{GmbH}$ 


\section{Autoren}

\section{Prof. Dr. sc.nat. Bernd Stark}

Technische Fachhochschule Wildau

Fachbereich Ingenieur-/Wirtschaftsingenieurwesen

Labor für Luftschadstoffmessung/Gasanalytik

Bahnhofstraße

15745 Wildau

Tel. (0 33 75) 504014

E-mail: bstark@igw.tfh-wildau.de

Dr. rer. nat. habil. Lothar Martini

Technische Fachhochschule Wildau

Fachbereich Ingenieur-/Wirtschaftsingenieurwesen

Labor für Luftschadstoffmessung/Gasanalytik

Bahnhofstraße

15745 Wildau

Tel. (0 33 75) 504014

E-mail: bstark@igw.tfh-wildau

\section{Dr.-Ing. Roland Wernecke}

deka Sensor+Technologie

Entwicklungs- und Vertriebsgesellschaft $\mathrm{mbH}$

Potsdamer Str. 18a

14513 Teltow

Tel. (0 33 28) 430250 